Article

\title{
Objective Understanding of Front-of-Package Nutrition Labels among Nutritionally At-Risk Individuals
}

\author{
Pauline Ducrot ${ }^{1, *}$, Caroline Méjean ${ }^{1}$, Chantal Julia ${ }^{1,2}$, Emmanuelle Kesse-Guyot ${ }^{1}$, \\ Mathilde Touvier ${ }^{1}$, Léopold K. Fezeu ${ }^{1}$, Serge Hercberg ${ }^{1,2}$ and Sandrine Péneau ${ }^{1}$ \\ ${ }^{1}$ Equipe de Recherche en Epidémiologie Nutritionnelle, Centre de Recherche en Epidémiologie et \\ Statistiques, Université Paris 13, Inserm (U1153), Inra (U1125), Cnam, COMUE Sorbonne Paris Cité, \\ Bobigny F-93017, France; E-Mails: c.mejean@eren.smbh.univ-paris13.fr (C.M.); \\ c.julia@eren.smbh.univ-paris13.fr (C.J.); e.kesse@eren.smbh.univ-paris13.fr (E.K.-G.); \\ m.touvier@eren.smbh.univ-paris13.fr (M.T.); 1.fezeu@eren.smbh.univ-paris13.fr (L.K.F.); \\ s.hercberg@eren.smbh.univ-paris13.fr (S.H.); s.peneau@eren.smbh.univ-paris13.fr (S.P.) \\ ${ }^{2}$ Département de Santé Publique, Hôpital Avicenne, Bobigny Cedex F-93017, France \\ * Author to whom correspondence should be addressed; E-Mail: p.ducrot@eren.smbh.univ-paris13.fr; \\ Tel.: +33-148-388-908; Fax: +33-148-388-931.
}

Received: 23 May 2015 / Accepted: 14 August 2015 / Published: 24 August 2015

\begin{abstract}
In the ongoing debate about front-of-package (FOP) nutrition labels, little data exist regarding nutritionally at-risk populations, although they are critical targets of prevention programs. This study aimed to compare the impact of FOP labels on the ability to rank products according to their nutritional quality among French adults potentially at risk of poor dietary quality $(N=14,230)$. Four labels were evaluated: Guideline Daily Amounts (GDA), Multiple Traffic Lights (MTL), 5-Color Nutrition Label (5-CNL), Green Tick (Tick), along with a reference without label. Mixed models were used to assess how individual characteristics and FOP labels were associated with the ability to rank products. Older participants and those with a lower educational level, income, nutritional knowledge, and likelihood of reading nutrition facts were less skilled at ranking food products according to nutritional quality. Compared with individual characteristics, nutrition labels had an increased impact on food product ranking ability. Overall, 5-CNL corresponded to the highest rate of correct responses, followed by MTL, GDA, and Tick $(p<0.0001)$. The strongest impact of 5-CNL was observed among individuals with no nutritional knowledge (odds ratio (OR): 20.24; 95\% confidence interval (CI): 13.19-31.06). Therefore, 5-CNL appeared to be effective at informing consumers, including those who are nutritionally at-risk, about the nutritional quality of food products.
\end{abstract}


Keywords: food labeling; front-of-package nutrition label; objective understanding; population at risk

\section{Introduction}

In the current fight against chronic diseases, promoting a healthy diet is a major objective of public health policies around the world [1]. One possible strategy for promoting a healthy diet is to encourage healthier food choices at the point of purchase [2]. However, in many western countries, the nutritional information currently displayed on food packages is generally difficult to read and interpret [3]. Thus, efforts should be made to provide simple and easily comprehensible information in order to enable consumers to make informed choices. For this reason, front-of-package (FOP) nutrition labeling is of major interest, since it increases consumer awareness of the nutritional quality of food [3-8].

Existing nutrition labels can be divided into two main categories: nutrient-specific labels and summary labels. Nutrient-specific labels display the amount of nutrients for which individual intake should be limited (e.g., fat, sodium). Examples of such labels include the Multiple Traffic Lights (MTL) used in the United Kingdom (UK) [9], and the Guideline Daily Amounts (GDA) used in the United States and in European countries, [10] now replaced by Reference Intakes (RI) in Europe [11]. In turn, summary labels provide the customer with an overall estimate of the nutritional quality of the product. Examples of such labels include simple formats, such as the Keyhole used in Scandinavian countries and applied only to healthy food products [12], and graded formats such as Guiding Stars used in North America, which provides a rating from zero to three stars according to the nutritional quality of the product [13].

A theoretical framework was proposed by Grunert et al., to describe consumer food choice mechanisms when confronted with nutrition labeling [5]. First, the label should retain consumer attention. Next, for use, the label must be accepted and understood. These steps are potentially influenced by the label format, but also by individual-level determinants such as age, educational level, and interest in and knowledge of nutrition [5]. Evidence in the literature suggests that color-coded labels were more effective at focusing consumer attention and were preferred by individuals of low socioeconomic status, low educational level and poor nutritional knowledge [3-6,14-16]. In addition, summary labels may be more helpful in guiding vulnerable consumers toward healthier food choices, as they are more easily understood compared with nutrient-specific labels [7,8,17-21]. However, recent reviews reported a lack of research with subgroups of the population who might be at increased risk of consuming a lower-quality diet and/or among those who are overweight or obese [3,6-8]. Such vulnerable population subgroups include the elderly [22], those of lower socio-economic status, lower educational level [23], and lower interest in and knowledge of nutrition [24]. To date, most studies assessing consumer understanding have been performed on small samples, hence preventing accurate evaluation of label impact across subgroups [14,18,25]. In addition, many of those studies used subjective measures of consumer understanding [14,19] or performed objective measurements based on the comparison of only two products, potentially leading to random responses [17,18,25-27].

Finally, only a few studies have evaluated the understanding of a graded summary label, whereas recent reviews have emphasized its promising effects in real-world settings [7,8]. Therefore, in the 
context of the ongoing debate about the most effective labeling format, it is important to compare the understanding of different FOP nutrition labels, including a graded format, in subgroups potentially at risk of making poor food choices and consuming a lower-quality diet.

The main purpose of this study which used a sample of French adults was to identify individuals with a reduced ability to rank products according to nutritional quality. In addition, the influence of FOP labels on consumers' ability to rank products according to nutritional quality was also assessed. Finally, the performance of each of four different FOP labels among nutritionally at-risk individuals was evaluated. The tested labels comprised two nutrient-specific formats (GDA and MTL) and two summary formats (Green Tick (Tick) and 5-Color Nutrition Label (5-CNL)).

\section{Materials and Methods}

\subsection{Population}

The NutriNet-Santé study is an ongoing web-based prospective cohort study launched in France in May 2009 with a scheduled follow-up period of 10 years. It aims to investigate the relationship between nutrition and chronic disease risk, as well as determinants of dietary behavior and nutritional status. The study was implemented in the general French population (Internet-using adult volunteers, aged $\geqslant 18$ years). The rationale, design and methodology of the study have been published elsewhere [28]. In brief, to be included in the study, participants complete a baseline set of self-administered Web-based questionnaires assessing dietary intake, physical activity, anthropometric characteristics, lifestyle, socioeconomic conditions, and health status. As part of the follow-up, participants are asked on an annual basis to complete the same set of questionnaires. In addition, participants receive monthly email invitations to complete questionnaires about determinants of eating behavior, health status, etc. The study is conducted in accordance with the Declaration of Helsinki and all procedures were approved by the Institutional Review Board of the French Institute for Health and Medical Research (IRB Inserm n ${ }^{\circ} 0000388$ FWA00005831) and the Commission Nationale de l'Informatique et des Libertés (CNIL ${ }^{\circ} 908450$ and $\mathrm{n}^{\circ}$ 909216). All participants provide informed consent with an electronic signature. This study is registered in EudraCT (n`2013-000929-31).

\subsection{Socio-Demographic Characteristics and Purchasing Habits Data}

At baseline and annually, thereafter, participants in the NutriNet-Santé study are asked to provide socio-demographic data, including sex, age (18-30, 30-50, 50-65, >65 years), educational level (up to secondary, some college or university degree), and income (<1200€, 1200-1800€, 1800-2700€ and $>2700 €$ per consumption unit). Monthly household income is calculated per "consumption unit" (CU), where one $\mathrm{CU}$ is attributed for the first adult in the household, $0.5 \mathrm{CU}$ for other persons aged 14 or older, and $0.3 \mathrm{CU}$ for children under 14, following national statistics methodology and guidelines [29]. For each participant, the most up-to-date available socio-demographic data were used.

With regard to nutritional knowledge, participants were asked to self-estimate and report their level by choosing one of four options, ranging from "I know quite a bit about nutrition" to "I don't know anything about nutrition". 
Purchasing habits data comprised information about frequency of reading the ingredient lists and/or nutrition facts (always, often, sometimes, never) on the packages, as well as grocery shopping frequency (always, often, sometimes, never).

\subsection{Design}

\subsubsection{Procedure}

Objective understanding of the different FOP labeling formats was assessed in July 2014 via a Web-based questionnaire, under five different conditions: four alternatives corresponding to the four different FOP label formats and one alternative with no label. Subjects were asked to rank three products belonging to the same food category (e.g., Figure 1) according to their nutritional quality. Specifically, participants were shown pictures of the three products, each featuring the respective FOP label, and were asked: "From your point of view, please rank these products according to their nutritional quality". For the ranking, participants could choose among the following options: "lowest nutritional quality", "intermediate nutritional quality", "highest nutritional quality", or "I don't know". The three products were selected based on their differing nutritional quality, thus enabling ranking via the labels (except for the Tick format which enabled distinguishing only the top quality product). No other information on nutritional facts was provided and all quality labels (e.g., organic certification) were removed from the product images.

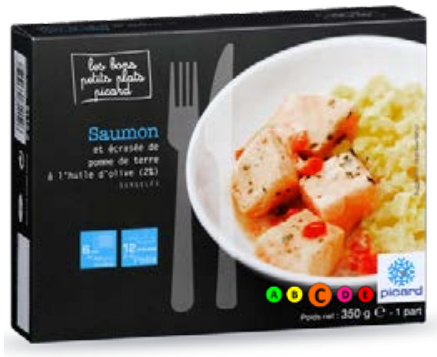

A-B-C-D-E

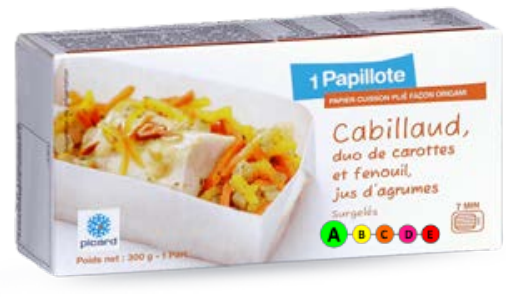

A-

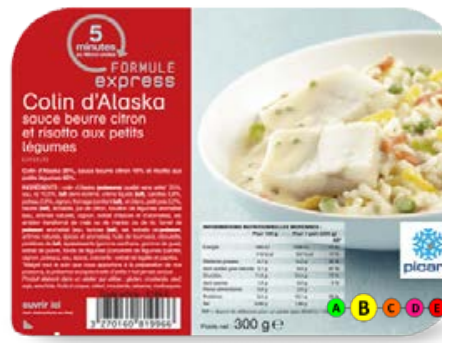

B

Figure 1. Screenshot of the stimulus material used in the study.

Five different product categories were tested: frozen prepared fish dishes, fresh pizzas, regular dairy products (mixed yogurts, cottage cheese and Greek yogurt), muesli breakfast cereals (chocolate, fruits and dried fruits/nuts), and appetizers (crisps and peanuts). To avoid potential effects of the product category upon understanding of the FOP label (i.e., due to knowledge of specific products), each label was associated with all product categories. Each participant was shown five label/product combinations where all five FOP label conditions and five product categories were represented. A rotation system based on a Latin Square design was employed to ensure that an equal number of participants were shown each label/product category combination while controlling for potential order effect of the labels. Thus, a total of 25 different versions of the questionnaire were used. For example, one participant was shown the 5-CNL on frozen prepared fish dishes, and MTL on fresh pizzas, while another participant was shown the 5-CNL on fresh pizzas and MTL on dairy products, etc. In addition, one respondent would be shown the 5-CNL first, while another participant would be shown the MTL first, etc. 
Ranking was considered correct if the three products were ranked in the expected order (i.e., according to information on nutritional quality provided by the labels). Ranking was considered as incorrect if at least one mistake was made, or if the answer "I don't know" was given. Expected ranking was the same whatever the situation.

\subsubsection{Label Formats}

As noted above, four different label formats (Figure 2), providing varying levels of information about the products' nutritional quality, were tested in the study. In the introduction to the questionnaire, the different label formats were presented and briefly explained to the participants.

Nutrient-specific formats:

1 Guideline Daily Amounts (GDA): this label indicates the kilocalories and the amount of total fat, saturated fatty acids, sugars, and sodium in grams per portion, as well as the corresponding contribution (in percentages) to the guideline-based daily intakes of these nutrients [10]. This label can be found on most food packaging on the French market, following a voluntary initiative on part of manufacturers. The GDA information was calculated by using the Food and Drink Federation's guiding principles and was based on the average nutrient requirements for an adult woman.

2 Multiple Traffic Lights (MTL): this label, introduced by the Food Standards Agency (FSA) in the UK, provides an evaluation of the nutrient content regarding total fat, saturated fatty acids, sugars, and sodium. Depending on the quantity of the specific nutrient in the product (high, medium, low), a color is attributed to each nutrient (red, amber, green) indicating the nutritional quality of the product. Healthier food products feature more green and fewer red codes. The colors reflect the concentration in grams per $100 \mathrm{~g}$ (or $100 \mathrm{~mL}$ ) of product, and the criteria of the FSA were applied to assign the color codes [9].

Summary formats:

3. Green Tick label (Tick): this label was derived from the "Keyhole" and "Pick the Tick" symbols, developed by the Swedish Food Administration and the Heart Foundation in Australia and New Zealand, respectively [12,30]. It reflects the overall nutritional quality of the food item and appears only on the healthier products within a food family. The Tick label was attributed to products assigned to the green or yellow categories by the 5-CNL (described below), and corresponds to "healthier" categories, as defined by the Office of Communication (OfCom) for advertising regulation [31].

4. Five-Color Nutrition Label (5-CNL): this label has been proposed specifically for the French market to guide consumer food choices [32]. It is based on the FSA nutrient profiling system [31], used by the OfCom. An adaptation of the guidelines was used specifically for cheese and added fat. This label provides information about the overall nutritional quality of a given food item. The label is represented by a scale of five colors, from green for the highest nutritional quality category to red for the lowest nutritional quality category, with corresponding letters (from A to E) [32]. Depending on the FSA score for each food item, the 5-CNL was: "green" (-15 to -2 points), "yellow" ( -1 to 3 points), “orange" (4 to 11 points), "pink" (12 to 16 points), and "red" ( $\geqslant 17$ points) [32]. 
Reference:

5. No label: a situation without any FOP nutrition labels was used as reference.

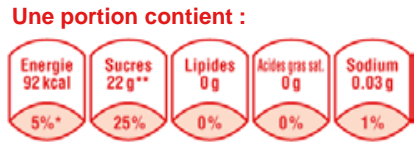

Guideline Daily Amounts

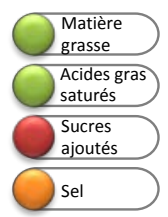

Multiple Traffic Lights

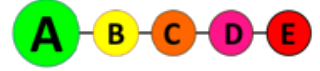

5 Color Nutrition Label

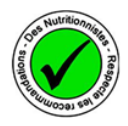

Green Tick

Figure 2. Front-Of-Package nutrition labels used in the study.

\subsection{Statistical Analysis}

Analysis was performed in 2015 on participants who had completed the questionnaire on FOP labels. Participants who had responded "I don't know" to more than two-thirds of the items were excluded from analysis. Chi-square tests were used to compare included and excluded subjects.

Mixed models for correlated data were used to evaluate how nutrition label formats and individual-level characteristics were associated with the ability to rank the three products. Individual characteristics were sex, age, educational level, monthly income, perceived nutritional knowledge, frequency of reading nutritional facts on product packages, and grocery shopping frequency. Variables displaying a significance level of $p<0.15$ in univariate models were retained for the multivariate model. Missing covariate data for educational level and income/CU were imputed using the multiple imputation method. Interactions between correlates of FOP label understanding were assessed. Due to significant interactions, the multivariate logistic regression model was used to perform stratified analyses according to the individual characteristics presented above. Sensitivity analyses were performed on the whole sample, without excluding any participants. All tests of significance were two-sided, and a $p$-value $<0.05$ was considered significant. Statistical analyses were performed using SAS software (version 9.3; SAS Institute Inc., Cary, NC, USA).

\section{Results}

\subsection{Characteristics of the Sample}

A total of 15,002 participants completed the FOP questionnaire. Among them, 772 were excluded because they had responded "I don't know" to more than two-thirds of the items, which left 14,230 participants available for analysis. Characteristics of included and excluded participants are presented in Table 1. Compared with excluded participants, included participants were more often women, younger, had a higher educational level and higher perceived nutritional knowledge; they were also more likely to read nutritional facts on food packages and to do grocery shopping. 
Table 1. Individual characteristics of included $(N=14,230)$ and excluded $(N=$ 772) participants.

\begin{tabular}{|c|c|c|c|c|c|}
\hline & \multicolumn{2}{|c|}{ Included $N=14,230$} & \multicolumn{2}{|c|}{ Excluded $N=772$} & \multirow{2}{*}{$p^{\text {a }}$} \\
\hline & $N$ & $\%$ & $N$ & $\%$ & \\
\hline \multicolumn{6}{|c|}{ Sex } \\
\hline Men & 3111 & 21.86 & 308 & 39.90 & $<0.0001$ \\
\hline Women & 11,119 & 78.14 & 464 & 60.10 & \\
\hline \multicolumn{6}{|c|}{ Age (year) } \\
\hline $18-30$ & 2121 & 14.72 & 13 & 1.68 & $<0.0001$ \\
\hline $30-50$ & 5112 & 14.91 & 164 & 21.24 & \\
\hline $50-65$ & 4903 & 35.92 & 336 & 43.52 & \\
\hline$>65$ & 2094 & 34.46 & 259 & 33.55 & \\
\hline \multicolumn{6}{|c|}{ Educational level } \\
\hline $\begin{array}{l}\text { Up to } \\
\text { secondary }\end{array}$ & 4335 & 30.46 & 354 & 45.85 & $<0.0001$ \\
\hline Some college & 4432 & 31.15 & 187 & 24.22 & \\
\hline $\begin{array}{l}\text { University } \\
\text { degree }\end{array}$ & 5203 & 36.56 & 198 & 25.65 & \\
\hline Missing data & 260 & 1.83 & 33 & 4.27 & \\
\hline \multicolumn{6}{|c|}{ Monthly income per household unit $\left(€ / \mathbf{C U}^{\mathbf{b}}\right)$} \\
\hline$<1200$ & 2089 & 14.68 & 112 & 14.51 & 0.25 \\
\hline $1200-1800$ & 3222 & 22.64 & 180 & 23.32 & \\
\hline $1800-2700$ & 3537 & 24.86 & 185 & 23.96 & \\
\hline$>2700$ & 3895 & 27.37 & 196 & 25.39 & \\
\hline Missing data & 1487 & 10.45 & 99 & 12.82 & \\
\hline \multicolumn{6}{|c|}{ Perceived nutritional knowledge level } \\
\hline High & 1966 & 13.82 & 72 & 9.33 & $<0.0001$ \\
\hline Medium & 7618 & 53.53 & 305 & 39.51 & \\
\hline Low & 4323 & 30.38 & 311 & 40.28 & \\
\hline $\begin{array}{l}\text { No } \\
\text { knowledge }\end{array}$ & 323 & 2.27 & 84 & 10.88 & \\
\hline
\end{tabular}

\begin{tabular}{lccccc}
\hline \multicolumn{5}{c}{ Frequency of reading nutritional facts on product packages } \\
\hline Always & 2913 & 20.47 & 149 & 19.30 & $<\mathbf{0 . 0 0 0 1}$ \\
Often & 7079 & 49.75 & 269 & 34.84 & \\
Sometimes & 3766 & 26.47 & 265 & 34.33 & \\
Never & 472 & 3.32 & 89 & 11.53 & \\
\hline \multicolumn{5}{c}{ Grocery shopping frequency } \\
\hline Always & 7965 & 55.97 & 399 & 51.68 & $<\mathbf{0 . 0 0 0 1}$ \\
Often & 4426 & 31.10 & 218 & 28.24 & \\
Sometimes & 1609 & 11.31 & 11 & 14.38 & \\
Never & 230 & 1.62 & 44 & 5.70 & \\
\hline
\end{tabular}

a: $p$-values based on chi-squared test; $\mathrm{CU}^{\mathrm{b}}$ : "consumption unit". One $\mathrm{CU}$ is attributed for the first adult in the household, 0.5 CU for other persons aged 14 or older, and $0.3 \mathrm{CU}$ for children under 14; Boldface indicates statistical significance $(p<0.05)$. 
3.2. Influence of Individual Characteristics and FOP Labels on the Ability to Rank Products according to Nutritional Quality

Results showing the association of product ranking ability with individual characteristics and label formats are presented in Table 2. Regarding socio-demographic characteristics, the odds of correctly ranking products were greater in women, younger participants, and those with a higher educational level. In addition, participants in the two highest income categories performed better in ranking products according to their nutritional quality compared with those in the lowest income category. Participants with the highest perceived nutritional knowledge gave more correct responses on the product ranking task than did those in the reference group, whereas those with medium-level knowledge of nutrition did not differ from the reference group. Similarly, participants who less frequently read nutritional facts on product packages gave less correct responses on the ranking task than did the reference, whereas those who reported often reading nutritional facts did not differ from the reference.

In both univariate and multivariate models, the odds of ranking products correctly according to nutritional quality was increased for all FOP labels used, compared with the no-label reference situation. Among the four formats, 5-CNL performed best, followed by MTL and GDA and the Tick label.

Odds ratios corresponding to the effect of FOP labels on ranking performance were far stronger than those corresponding to the effect of individual characteristics.

Sensitivity analyses performed on the whole sample, without exclusion of participants, showed similar trends.

Table 2. Univariate and multivariate analyses based on mixed models showing the association between Front-of-Package label formats and objective understanding $(N=14,230)^{\text {a }}$.

\begin{tabular}{|c|c|c|c|c|}
\hline & Univariate & \multirow{2}{*}{$p$} & Mutivariate & \multirow{2}{*}{$p$} \\
\hline & OR $(95 \%$ CI $)$ & & OR $(95 \% \mathrm{CI})$ & \\
\hline \multicolumn{5}{|c|}{ FOP labels } \\
\hline No label & 1 & & 1 & \\
\hline 5-CNL & $12.07(11.41-12.78)$ & $<0.0001$ & $12.61(11.91-13.36)$ & $<0.0001$ \\
\hline MTL & $8.38(7.92-8.86)$ & $<0.0001$ & $8.71(8.22-9.22)$ & $<0.0001$ \\
\hline GDA & $7.47(7.06-7.91)$ & $<0.0001$ & $7.74(7.31-8.20)$ & $<0.0001$ \\
\hline Tick & $2.34(2.21-2.47)$ & $<0.0001$ & $2.36(2.23-2.49)$ & $<0.0001$ \\
\hline \multicolumn{5}{|c|}{ Sex } \\
\hline Men & 1 & & 1 & \\
\hline Women & $1.22(1.18-1.27)$ & $<0.0001$ & $1.12(1.06-1.17)$ & $<0.0001$ \\
\hline \multicolumn{5}{|c|}{ Age (year) } \\
\hline $18-30$ & 1 & & 1 & \\
\hline $30-50$ & $0.92(0.88-0.96)$ & $<0.0001$ & $0.88(0.83-0.93)$ & $<0.0001$ \\
\hline $50-65$ & $0.69(0.66-0.73)$ & $<0.0001$ & $0.64(0.61-0.68)$ & $<0.0001$ \\
\hline$>65$ & $0.53(0.50-0.56)$ & $<0.0001$ & $0.47(0.44-0.51)$ & $<0.0001$ \\
\hline
\end{tabular}


Table 2. Cont.

\begin{tabular}{|c|c|c|c|c|}
\hline \multicolumn{5}{|c|}{ Educational level } \\
\hline Up to secondary & 1 & & 1 & \\
\hline Some college & $1.24(1.19-1.29)$ & $<0.0001$ & $1.15(1.10-1.21)$ & $<0.0001$ \\
\hline $\begin{array}{l}\text { University } \\
\text { degree }\end{array}$ & $1.30(1.25-1.35)$ & $<0.0001$ & $1.17(1.11-1.22)$ & $<0.0001$ \\
\hline \multicolumn{5}{|c|}{ Monthly income per household unit $\left(€ / \mathbf{C U}^{b}\right)$} \\
\hline$<1200$ & 1 & & 1 & \\
\hline $1200-1800$ & $0.94(0.90-0.99)$ & 0.033 & $1.00(0.94-1.07)$ & 0.99 \\
\hline $1800-2700$ & $0.99(0.94-1.04)$ & 0.74 & $1.09(1.02-1.16)$ & 0.0068 \\
\hline$>2700$ & $0.99(0.94-1.04)$ & 0.65 & $1.11(1.05-1.18)$ & 0.0008 \\
\hline \multicolumn{5}{|c|}{ Perceived nutritional knowledge level } \\
\hline High & 1.00 & & 1 & \\
\hline Medium & $0.92(0.88-0.96)$ & 0.0004 & $0.99(0.94-1.05)$ & 0.84 \\
\hline Low & $0.81(0.77-0.85)$ & $<0.0001$ & $0.92(0.86-0.98)$ & 0.0072 \\
\hline No knowledge & $0.69(0.61-0.77)$ & $<0.0001$ & $0.81(0.71-0.93)$ & 0.0022 \\
\hline \multicolumn{5}{|c|}{ Frequency of reading nutritional facts on product packages } \\
\hline Always & 1 & & 1 & \\
\hline Often & $0.98(0.94-1.02)$ & 0.35 & $0.99(0.94-1.04)$ & 0.69 \\
\hline Sometimes & $0.89(0.85-0.93)$ & $<0.0001$ & $0.90(0.85-0.95)$ & 0.0004 \\
\hline Never & $0.77(0.70-0.85)$ & $<0.0001$ & $0.77(0.68-0.87)$ & $<0.0001$ \\
\hline \multicolumn{5}{|c|}{ Grocery shopping frequency } \\
\hline Always & 1 & & 1 & \\
\hline Often & $1.03(0.99-1.06)$ & 0.12 & $1.00(0.96-1.04)$ & 0.95 \\
\hline Sometimes & $0.94(0.89-0.99)$ & 0.015 & $1.02(0.96-1.09)$ & 0.56 \\
\hline Never & $0.83(0.73-0.94)$ & 0.0039 & $0.93(0.80-1.09)$ & 0.38 \\
\hline
\end{tabular}

a: The modeled probability was correct ranking of the three products according to their nutritional quality; CU

b: "consumption unit". One CU is attributed for the first adult in the household. 0.5 CU for other persons aged 14 or older, and $0.3 \mathrm{CU}$ for children under 14; Boldface indicates statistical significance $(p<0.05)$; OR:odds ratio; CI: confidence interval; FOP: front-of-package.

\subsection{Comparison of Label Performance across Subgroups}

Label performances across subgroups of individuals are presented in Table 3. Results show the same trend across subgroups, although odds ratios vary to differing extents. Compared to the reference situation (no label), all label formats significantly increased the ability of participants to correctly rank products according to nutritional quality. The 5-CNL was the label that had the best performance in all subgroups (lowest OR: 8.72, (95\% CI: 7.46-10.18); greatest OR: 20.24 (95\% CI: 12.59-31.06)), followed by MTL (OR range: 6.30 (5.39-7.35)-10.62 (9.15-12.33), GDA (OR range: 5.53 (3.99-7.66)-8.79 (7.92-9.76)) and Tick (OR range: 1.98 (1.72-2.29)- 2.64 (1.63-4.27)) labels.

Sensitivity analyses performed on the whole sample, with no exclusion of participants, revealed similar trends. 
Table 3. Multivariate mixed model of the association between objective understanding and Front-of-Package labels, across subgroups at risk $(N=14,230)^{\text {a }}$.

\begin{tabular}{|c|c|c|c|c|c|c|}
\hline & $\begin{array}{c}\text { No } \\
\text { Label }\end{array}$ & $\frac{\text { 5-CNL }}{\text { OR (CI 95\%) }}$ & $\frac{\text { MTL }}{\text { OR (CI 95\%) }}$ & $\frac{\text { GDA }}{\text { OR (CI 95\%) }}$ & $\begin{array}{c}\text { Tick } \\
\text { OR (CI 95\%) }\end{array}$ & $p^{b}$ \\
\hline \multicolumn{7}{|c|}{ Sex } \\
\hline Men & 1 & $13.44(11.83-15.27)$ & $9.33(8.21-10.60)$ & $7.45(6.56-8.46)$ & $2.55(2.24-2.90)$ & $<0.0001$ \\
\hline Women & 1 & $12.41(11.63-13.24)$ & $8.55(8.02-9.12)$ & $7.85(7.36-8.37)$ & $2.31(2.17-2.46)$ & $<0.0001$ \\
\hline \multicolumn{7}{|c|}{ Age (year) } \\
\hline $18-30$ & 1 & $14.20(12.25-16.46)$ & $10.62(9.15-12.33)$ & $8.56(7.41-9.89)$ & $2.28(1.99-2.61)$ & $<0.0001$ \\
\hline $30-50$ & 1 & $15.36(13.93-16.94)$ & $9.74(8.85-10.71)$ & $8.05(7.32-8.86)$ & $2.39(2.18-2.62)$ & $<0.0001$ \\
\hline $50-65$ & 1 & $11.50(10.43-12.68)$ & $8.03(7.28-8.85)$ & $7.36(6.67-8.11)$ & $2.32(2.10-2.56)$ & $<0.0001$ \\
\hline$>65$ & 1 & $8.72(7.46-10.18)$ & $6.30(5.39-7.35)$ & $6.63(5.68-7.74)$ & $2.40(2.04-2.82)$ & $<0.0001$ \\
\hline \multicolumn{7}{|c|}{ Educational level } \\
\hline Up to secondary & 1 & $9.91(8.94-11.00)$ & $7.30(6.58-8.10)$ & $7.03(6.34-7.80)$ & $2.44(2.20-2.72)$ & $<0.0001$ \\
\hline Secondary & 1 & $12.59(11.36-13.96)$ & 8.73 (7.88-9.66) & $7.26(6.55-8.05)$ & $2.39(2.16-2.64)$ & $<0.0001$ \\
\hline University & 1 & $15.61(14.17-17.19)$ & $10.00(9.09-11.01)$ & 8.77 (7.98-9.64) & $2.26(2.06-2.48)$ & $<0.0001$ \\
\hline \multicolumn{7}{|c|}{ Monthly income per household unit $\left(€ / \mathbf{C U}^{\mathbf{c}}\right)$} \\
\hline$<1200$ & 1 & $11.99(10.42-13.79)$ & $8.37(7.24-9.66)$ & $7.22(6.27-8.31)$ & $2.33(2.03-2.69)$ & $<0.0001$ \\
\hline $1200-1800$ & 1 & $12.46(11.09-14.00)$ & $8.79(7.82-9.87)$ & $7.59(6.75-8.54)$ & $2.54(2.26-2.85)$ & $<0.0001$ \\
\hline $1800-2700$ & 1 & $11.84(10.56-13.28)$ & $7.89(7.04-8.85)$ & $7.48(6.66-8.40)$ & $2.12(1.90-2.38)$ & $<0.0001$ \\
\hline$>2700$ & 1 & $14.06(12.59-15.71)$ & $9.77(8.76-10.90)$ & $8.54(7.65-9.53)$ & $2.47(2.22-2.74)$ & $<0.0001$ \\
\hline \multicolumn{7}{|c|}{ Perceived nutritional knowledge level } \\
\hline High & 1 & $10.52(9.05-12.22)$ & $7.79(6.71-9.04)$ & $7.43(6.40-8.62)$ & $1.98(1.72-2.29)$ & $<0.0001$ \\
\hline Medium & 1 & $12.46(11.53-13.47)$ & $8.74(8.08-9.45)$ & $8.54(7.89-9.24)$ & $2.38(2.21-2.57)$ & $<0.0001$ \\
\hline Low & 1 & $13.70(12.30-15.26)$ & $9.15(8.22-10.19)$ & $6.87(6.17-7.64)$ & $2.52(2.26-2.80)$ & $<0.0001$ \\
\hline No knowledge & 1 & $20.24(13.19-31.06)$ & $9.80(6.48-14.80)$ & $6.56(4.29-10.03)$ & $2.55(1.63-3.99)$ & $<0.0001$ \\
\hline
\end{tabular}


Table 3. Cont.

\begin{tabular}{ccccccc}
\hline \multicolumn{7}{c}{ Frequency of reading nutritional facts on product packages } \\
\hline Always & 1 & $10.32(9.14-11.64)$ & $8.00(7.07-9.06)$ & $8.02(7.09-9.06)$ & $2.23(1.98-2.52)$ & $<\mathbf{0 . 0 0 0 1}$ \\
Often & 1 & $13.14(12.10-14.26)$ & $9.08(8.37-9.86)$ & $8.58(7.90-9.31)$ & $2.48(2.29-2.69)$ & $<\mathbf{0 . 0 0 0 1}$ \\
Sometimes & 1 & $13.84(12.33-15.53)$ & $8.86(7.91-9.92)$ & $6.56(5.85-7.34)$ & $2.20(1.97-2.46)$ & $<\mathbf{0 . 0 0 0 1}$ \\
Never & 1 & $11.93(8.67-16.41)$ & $7.07(5.12-9.77)$ & $5.53(3.99-7.66)$ & $2.60(1.87-3.61)$ & $<\mathbf{0 . 0 0 0 1}$ \\
\hline \multicolumn{7}{c}{ Grocery shopping frequency } \\
\hline Always & 1 & $11.41(10.58-12.32)$ & $7.93(7.36-8.56)$ & $7.25(6.72-7.82)$ & $2.30(2.14-2.48)$ & $<\mathbf{0 . 0 0 0 1}$ \\
Often & 1 & $14.69(13.22-16.32)$ & $9.68(8.71-10.76)$ & $8.79(7.92-9.76)$ & $2.39(2.16-2.65)$ & $<\mathbf{0 . 0 0 0 1}$ \\
Sometimes & 1 & $14.06(11.80-16.74)$ & $10.47(8.78-12.48)$ & $7.89(6.62-9.42)$ & $2.55(2.14-3.04)$ & $<\mathbf{0 . 0 0 0 1}$ \\
Never & 1 & $12.92(8.06-20.70)$ & $9.52(5.92-15.31)$ & $7.11(4.50-11.23)$ & $2.64(1.63-4.27)$ & $<\mathbf{0 . 0 0 0 1}$ \\
\hline
\end{tabular}

${ }^{a}$ Model was adjusted for sex, age, educational level, monthly income, purchasing frequency, perceived nutritional knowledge and frequency of reading nutritional facts on product packages; ${ }^{\mathrm{b}}$ The modeled probability was a correct ranking of the three products according to their nutritional quality; ${ }^{\mathrm{c}} \mathrm{CU}$ : consumption units. One $\mathrm{CU}$ is attributed for the first adult in the household. 0.5 for other persons aged 14 or older and 0.3 for children under 14; Boldface indicates statistical significance $(p<0.05)$; GDA: Guideline Daily Amounts; MTL: Multiple Traffic Lights; 5-CNL: 5-Color Nutrition Label; Tick: Green Tick; OR:odds ratio; CI: confidence interval. 


\section{Discussion}

Results of the present study indicate that understanding of FOP labels differs across groups of individuals. The ability to rank products according to nutritional quality was lower in older subjects, men, participants with a lower educational level, lower income, lower nutritional knowledge, and those who were less likely to read nutrition facts on food packages. However, results of the present study also indicated that, compared to individual characteristics, nutrition labels had a stronger influence on food product ranking ability. The 5-CNL graded summary label was the format that was most easily understood in all subgroups, and it performed particularly well among participants with no nutritional knowledge.

\subsection{Influence of Individual Characteristics and FOP Labels on the Ability to Rank Products according to Nutritional Quality}

Results of the present study are in line with previous reports that found that some population subgroups display a decreased likelihood of understanding FOP nutrition labels $[3,7,8,15,17,18]$. In this study, women performed significantly better than did men. Women's greater interest in nutrition [5], as well as the fact that they are more likely to use nutrition labels [3-5,33], might account for their increased ability to rank products according to nutritional quality. Data from the present study support previous studies indicating that older participants and those with lower educational level had more difficulty in understanding nutrition labels $[14,15,17,26,34]$. Similar to this study, two studies indicated that participants with lower self-reported nutritional knowledge or who less often read labels were less likely to accurately interpret FOP labels $[14,18]$. To our knowledge, no study has evaluated the impact of income on FOP label understanding. However, two studies reported that consumers belonging to lower socio-economic strata had more difficulty understanding nutrition labeling than did their more affluent counterparts $[17,18]$. Thus, the positive association between income and understanding of FOP labels, which was observed in the present study following adjustment for educational level, could be due to social status, potentially influencing the interest in healthy eating, and the ability to process information [15].

Overall, in the present study, participants displaying increased difficulty understanding nutrition labels had similar sociodemographic profiles to individuals displaying a reduced likelihood of using nutrition labels $[3,4,7]$, and those potentially at-risk regarding their nutritional status [35-41]. Generally, a better understanding of labels promotes their use [42], thus, it appears crucial to select FOP labels that enable accurate understanding among vulnerable subgroups.

Consistent with previous work, results of the present study indicate that nutrition labels are efficient tools for increasing consumers' ability to compare nutritional quality across food products, compared with a reference situation presenting no label [7,17,18,25,27,43]. Among the different label formats, 5-CNL performed best at enabling consumers to rank food products according nutritional quality, followed by MTL, GDA, and the Tick label, which supports the potential of a graded summary label suggested in recent reviews [7,8].

Interestingly, results of this study highlighted the fact that nutrition labels improve individuals' ability to rank food products according to nutritional quality to a much greater extent (greatest OR: 12.64 
(95\%CI: 11.93-13.39)), than did individual characteristics (greatest OR: 1.17 (95\%CI: 1.12-1.23)). This is of particular interest given that nutrition labeling is conceivably easier to modify than are individual factors. Among potentially-modifiable individual-level factors, nutritional knowledge and the frequency of reading nutrition facts on food packages could be improved by nutrition education $[44,45]$. However, such interventions are most effective when dispensed in childhood [46], with a potential impact over the long term. In turn, previous studies have suggested that introducing nutrition labels had the advantage of being cost-effective and could bring about substantial and relatively quick health benefits on the population level $[47,48]$.

\subsection{Comparison of Label Performance across Subgroups}

Across subgroups of participants, the 5-CNL had the strongest positive association with participants' ability to rank products, followed by MTL, GDA and Tick. This trend was observed especially in subjects with low nutritional knowledge. Although several reviews emphasized the potential of a graded label $[7,8]$, consumer understanding of such a format has been poorly studied in the literature. To our knowledge, only one study found that graded labels were easier to understand than was the color-coded GDA among participants with poor nutritional knowledge and who never read labels [14]. However, no significant differences were observed between the summary graded label and MTL and label formats were similarly understood in individuals of various age and educational level [14]. A possible explanation for such discrepancies might be that the large sample size used in the present study provided sufficient statistical power for performing subgroup analyses. Thus, a more accurate comparison among label formats was possible. Next, two particular attributes might explain the better performance of 5-CNL compared with MTL. First, 5-CNL summarizes the product's nutritional quality in a single indicator, thus preventing misunderstanding of nutrition terminology [4] and obviating the need to process and synthesize information on nutrient content. Second, the 5-CNL combined color and text, which has been shown to improve readability of FOP labels [6,7]. The 5-CNL outperformed all other formats across all nutritionally at-risk subgroups and performed particularly well among individuals with no nutritional knowledge. Thus, this format appeared to be a well-adapted tool for fairly informing consumers about the nutritional quality of foods.

As regards the nutrient-specific label formats, and in agreement with previous research, results of the present study showed that the MTL was better than the GDA at increasing understanding $[17-19,25,26]$. Indeed, color-coded labels such as MTL have been shown to enhance consumer ability to evaluate product healthiness [7]. In turn, nutrition labels that include numbers and percentages, such as GDA, were found to be confusing to many consumers, particularly those with lower educational levels or literacy $[3,6,7]$. In contrast with these findings, results of a recent study performed among consumers from the UK, Germany, Poland, and Turkey showed that GDA and MTL labels including numerical nutritional information performed similarly to enable consumers to identify the healthier alternative within a set of three food products [49]. A possible explanation to account for such discrepancies may be that numbers displayed on the MTL have been confusing for participants. Although previous studies reported that simple summary formats are preferred by vulnerable subgroups [14,19,20,50], in the present study, the Tick label was associated with the poorest performance as regards consumer understanding. However, Malam et al. noted that the preference for a particular FOP labeling format 
did not necessarily indicate a better understanding of that label [18]. In addition, in a previous study, a simple summary label showed similar effect compared with other FOP labels (i.e., GDA, MTL), at differentiating healthiness of three food products [49], suggesting that such a format might not necessarily be optimal when ranking more than two products according to their nutritional quality. One explanation to account for the poor performance of the Tick label might be that such a format enables consumers to identify healthier products, but not to differentiate between products of medium and low nutritional quality $[51,52]$. Thus, oversimplification might lead to a loss of information and to misinterpretation of the label $[53,54]$.

\subsection{Strengths and Limitations}

A major strength of this study was its large sample size and the heterogeneity of socio-demographic profiles, enabling sufficiently-powered analyses across subgroups. Moreover, to limit desirability bias, an objective measure of participant understanding was used. A set of three products was used so as to be more realistic and to limit random responses. In addition, the potential effects of the labels' order of appearance were controlled by using a rotation system. To limit the effect related to product choice, five food categories were included and all combinations of products/labels were tested.

This study is subject to several limitations. First, participants of the NutriNet-Santé study are volunteers in a nutrition-focused cohort. Compared with excluded participants, included participants had higher knowledge of nutrition and read nutrition facts more often. Indeed, compared with the present study, previous studies reported lower levels of nutrition facts reading among French consumers (i.e., 9\%) [55] and lower knowledge of nutrition guidelines across a representative sample of French adults [56]. Caution is, therefore, needed when interpreting and generalizing the present results. However, interest in, and knowledge of, nutrition might have led to a greater number of correct responses in the reference situation ("no-label") compared with the general French population, thus possibly decreasing the labels' influence. In addition, the study was performed among Internet users, possibly leading to sociodemographic differences compared with the general French population [57]. However, the sample featured sociodemographic diversity, including individuals from typically under-represented subgroups in traditional surveys (older, low socio-economic status). Another limitation pertained to the fact that the assessment was not performed in a real-life environment, where a number of factors influence consumer understanding. Indeed, noise, marketing messages, time pressure, and the multitude of available products are likely to hinder label processing [58-60].

\section{Conclusions}

The present study is one of the first investigating label understanding in a large sample of general population-based volunteers, including individuals potentially at at-risk regarding their nutritional status. The results showed that the ability to compare products according to nutritional quality differed across groups of individuals and was lower in nutritionally at-risk individuals. The results also highlighted the fact that the impact of nutrition labeling on product comparison was stronger than were individual characteristics. These data bring new supportive evidence to the current debate on food labeling. In particular, the 5-CNL graded color-coded label displayed a strong performance across population subgroups. In addition, this graded label was shown to perform particularly well in participants with no 
nutritional knowledge. Introducing such a label would provide a relatively fair understanding of FOP labels among consumers, and might potentially encourage those who do not frequently use nutritional information to favorably change their behavior as regards food choices.

\section{Acknowledgments}

We thank all the scientists, dietitians, technicians and assistants who help carry out the NutriNet-Santé study. We especially thank Younes Esseddik, Yasmina Chelghoum, Mohand Ait Oufella, Paul Flanzy and Thi Hong Van Duong, computer scientists; Veronique Gourlet, Charlie Menard, Fabien Szabo, Nathalie Arnault, Laurent Bourhis and Stephen Besseau, statisticians; and the dietitians. We are grateful to the volunteers of the NutriNet-Santé study.

We thank the French Ministry of Health (DGS) and the French National Institute for Prevention and Health Education (INPES) for supporting this project.

The NutriNet-Santé Study is supported by the French Ministry of Health (DGS), the French Institute for Public Health Surveillance (InVS), the French National Institute for Health and Medical Research (INSERM), the French National Institute for Agricultural Research (INRA), the National Conservatory for Arts and Crafts (CNAM), the National Institute for Prevention and Health Education (INPES) and the University of Paris 13.

\section{Author Contributions}

The authors' responsibilities were as follows: Pauline Ducrot: conducted the literature review, performed analyses and drafted the manuscript; Caroline Méjean, Chantal Julia, Emmanuelle Kesse-Guyot, Mathilde Touvier, Léopold K. Fezeu, Serge Hercberg and Sandrine Péneau: involved in the interpretation of results and critically reviewed the manuscript; and Serge Hercberg and Sandrine Péneau: were responsible for developing the study design and protocol.

\section{Conflicts of Interest}

The authors declare no conflict of interest.

\section{References}

1. World Health Organization. Diet, Nutrition and the Prevention of Chronic Diseases. In WHO Technical Report Series; 916; WHO: Geneva, Switzerland, 2003.

2. World Health Organization. Global Strategy on Diet, Physical Activity and Health; WHO: Geneva, Switzerland, 2004.

3. Campos, S.; Doxey, J.; Hammond, D. Nutrition labels on pre-packaged foods: A systematic review. Public Health Nutr. 2011, 14, 1496-1506. [CrossRef] [PubMed]

4. Cowburn, G.; Stockley, L. Consumer understanding and use of nutrition labelling: A systematic review. Public Health Nutr. 2005, 8, 21-28. [CrossRef] [PubMed]

5. Grunert, K.G.; Wills, J.M. A review of European research on consumer response to nutrition information on food labels. J. Public Health 2007, 15, 385-399. [CrossRef] 
6. Hawley, K.L.; Roberto, C.A.; Bragg, M.A.; Liu, P.J.; Schwartz, M.B.; Brownell, K.D. The science on front-of-package food labels. Public Health Nutr. 2013, 16, 430-439. [CrossRef] [PubMed]

7. Hersey, J.C.; Wohlgenant, K.C.; Arsenault, J.E.; Kosa, K.M.; Muth, M.K. Effects of front-of-package and shelf nutrition labeling systems on consumers. Nutr. Rev. 2013, 71, 1-14. [CrossRef] [PubMed]

8. Institute of Medicine. Front-of-Package Nutrition Rating Systems and Symbols: Promoting Healthier Choices; The National Academies Press: Washington, DC, USA, 2012.

9. Food Standards Agency. Front-of-pack Traffic light signpost labelling Technical Guidance; Food Standards Agency: Kingsway, UK, 2007.

10. Food and Drink Federation. GDAs Explained: Guideline Daily Amounts. Available online: http://www.gdalabel.org.uk/gda/gda_values.aspx (accessed on 29 October 2014).

11. Food and Drink Federation. References Intakes (previously Guideline Daily Amounts). Available online: http://www.foodlabel.org.uk/label/reference-intakes.aspx (accessed on 15 July 2015).

12. Swedish National Food Agency. Rules for the Keyhole Symbol. Available online: http://www.slv.se/en-gb/Group1/Food-and-Nutrition/Keyhole-symbol/Rules-for-the-keyhole-symbol/ (accessed on 1 December 2014).

13. Fischer, L.M.; Sutherland, L.A.; Kaley, L.A.; Fox, T.A.; Hasler, C.M.; Nobel, J.; Kantor, M.A.; Blumberg, J. Development and implementation of the guiding stars nutrition guidance program. Am. J. Health Promot. 2011, 26, e55-e63. [CrossRef] [PubMed]

14. Feunekes, G.I.; Gortemaker, I.A.; Willems, A.A.; Lion, R.; van den Kommer, M. Front-of-pack nutrition labelling: Testing effectiveness of different nutrition labelling formats front-of-pack in four European countries. Appetite 2008, 50, 57-70. [CrossRef] [PubMed]

15. Grunert, K.G.; Fernandez-Celemin, L.; Wills, J.M.; Storcksdieck Genannt, B.S.; Nureeva, L. Use and understanding of nutrition information on food labels in six European countries. Z. Gesundh. Wiss. 2010, 18, 261-277. [CrossRef] [PubMed]

16. Mejean, C.; Macouillard, P.; Peneau, S.; Hercberg, S.; Castetbon, K. Perception of front-of-pack labels according to social characteristics, nutritional knowledge and food purchasing habits. Public Health Nutr. 2013, 16, 392-402. [CrossRef] [PubMed]

17. Food Standards Agency. Quantitative Evaluation of Alternative Food Signposting Concepts: Report of Findings; Synovate: London, UK, 2005.

18. Malam, S.; Clegg, S.; Kirwan, S.; McGinigal, S. Comprehension and Use of UK Nutrition Signpost Lablling Schemes; University of Surrey: Guildford, UK, 2009.

19. Mejean, C.; Macouillard, P.; Peneau, S.; Hercberg, S.; Castetbon, K. Consumer acceptability and understanding of front-of-pack nutrition labels. J. Hum. Nutr. Diet. 2013, 26, 494-503. [CrossRef] [PubMed]

20. Mejean, C.; Macouillard, P.; Peneau, S.; Lassale, C.; Hercberg, S.; Castetbon, K. Association of perception of front-of-pack labels with dietary, lifestyle and health characteristics. PLoS ONE 2014, 9, e90971. [CrossRef] [PubMed] 
21. Ruffieux, B.; Muller, L. Etude sur L'influence de Divers Systèmes D'étiquetage Nutritionnel sur la Composition du Panier D'achat Alimentaire. Available online: http://agris.fao.org/ agris-search/search.do?recordID=FR2014008893 (accessed on 20 October 2014).

22. World Health Organization. Obesity and Overweight. Available online: http://www.who.int/ dietphysicalactivity/media/en/gsfs_obesity.pdf (accessed on 15 July 2015).

23. World Health Organization. Obesity and Inequities-Guidance for Addressing Inequities in Overweight and Obesity. Available online: http://www.euro.who.int/en/data-and-evidence/ equity-in-health-project/policy-briefs/obesity-and-inequities.-guidance-for-addressing-inequities-in -overweight-and-obesity-2014 (accessed on 15 January 2015).

24. Beydoun, M.A.; Powell, L.M.; Wang, Y. Reduced away-from-home food expenditure and better nutrition knowledge and belief can improve quality of dietary intake among US adults. Public Health Nutr. 2009, 12, 369-381. [CrossRef] [PubMed]

25. Borgmeier, I.; Westenhoefer, J. Impact of different food label formats on healthiness evaluation and food choice of consumers: A randomized-controlled study. BMC Public Health 2009, 9, 184. [CrossRef] [PubMed]

26. Kelly, B.; Hughes, C.; Chapman, K.; Louie, J.C.; Dixon, H.; Crawford, J.; King, L.; Daube, M.; Slevin, T. Consumer testing of the acceptability and effectiveness of front-of-pack food labelling systems for the Australian grocery market. Health Promot. Int. 2009, 24, 120-129. [CrossRef] [PubMed]

27. Roberto, C.A.; Bragg, M.A.; Seamans, M.J.; Mechulan, R.L.; Novak, N.; Brownell, K.D. Evaluation of consumer understanding of different front-of-package nutrition labels, 2010-2011. Prev. Chronic Dis. 2012, 9, E149. [CrossRef] [PubMed]

28. Hercberg, S.; Castetbon, K.; Czernichow, S.; Malon, A.; Mejean, C.; Kesse, E.; Touvier, M.; Galan, P. The Nutrinet-Sante Study: A web-based prospective study on the relationship between nutrition and health and determinants of dietary patterns and nutritional status. BMC Public Health. 2010, 10, 242. [CrossRef] [PubMed]

29. INSEE (Institut National de la Statistique et des Etudes Economiques). Consumption Unit Definition. Available online: http://www.insee.fr/en/methodes/default.asp?page=definitions/ unite-consommation.htm (accessed on 30 September 2014).

30. Heart Foundation. Tick Nutrient Criteria. Available online: http://www.heartfoundation.org.nz/ programmes-resources/food-industry-and-hospitality/tick-programme/tick-criteria (accessed on 29 October 2014).

31. Rayner, M.; Scarborough, P.; Lobstein, T. The UK Ofcom Nutrient Profiling Model—Defining "Healthy" and "Unhealthy" Food and Drinks for TV Advertising to Children. Available online: http://www.ndph.ox.ac.uk/bhfcpnp/about/publications-and-reports/group-reports/ uk-ofcom-nutrient-profile-model.pdf (accessed on 10 October 2014).

32. Hercberg, S. Propositions Pour un Nouvel élan de la Politique Nutritionnelle Française de Santé Publique Dans le cadre de la Stratégie Nationale de Santé. 1ère Partie: Mesures Concernant la Prévention Nutritionnelle. Available online: http://sante.gouv.fr/ IMG/pdf/rapport_Basdevant_15_11_2013.pdf (accessed on 15 October 2014). 
33. Drichoutis, A. Consumers' use of nutritional labels: A review of research studies and issues. Acad. Mark. Sci. Rev. 2006, 103, 93-118.

34. Scott, V.; Worsley, A.F. Ticks, claims, tables and food groups: A comparison for nutrition labelling. Health Promot. Int. 1994, 9, 27-37. [CrossRef]

35. Alkerwi, A.; Sauvageot, N.; Nau, A.; Lair, M.L.; Donneau, A.F.; Albert, A.; Guillaume, M. Population compliance with national dietary recommendations and its determinants: Findings from the ORISCAV-LUX study. Br. J. Nutr. 2012, 108, 2083-2092. [CrossRef] [PubMed]

36. Aranceta, J.; Perez-Rodrigo, C.; Serra-Majem, L.; Ribas, L.; Quiles-Izquierdo, J.; Vioque, J.; Foz, M.; Spanish Collaborative Group for the Study of Obesity. Influence of sociodemographic factors in the prevalence of obesity in Spain. The SEEDO'97 Study. Eur. J. Clin. Nutr. 2001, 55, 430-435. [CrossRef] [PubMed]

37. Beydoun, M.A.; Wang, Y. Do nutrition knowledge and beliefs modify the association of socio-economic factors and diet quality among US adults? Prev. Med. 2008, 46, 145-153. [CrossRef] [PubMed]

38. Gallus, S.; Odone, A.; Lugo, A.; Bosetti, C.; Colombo, P.; Zuccaro, P.; La Vecchia, C. Overweight and obesity prevalence and determinants in Italy: An update to 2010. Eur. J. Nutr. 2013, 52, 677-685. [CrossRef] [PubMed]

39. Harrington, J.; Fitzgerald, A.P.; Layte, R.; Lutomski, J.; Molcho, M.; Perry, I.J. Sociodemographic, health and lifestyle predictors of poor diets. Public Health Nutr. 2011, 14, 2166-2175. [CrossRef] [PubMed]

40. Lassale, C.; Galan, P.; Castetbon, K.; Peneau, S.; Mejean, C.; Hercberg, S.; Kesse-Guyot, E. Differential association between adherence to nutritional recommendations and body weight status across educational levels: A cross-sectional study. Prev. Med. 2013, 57, 488-493. [CrossRef] [PubMed]

41. Thiele, S.; Mensink, G.B.; Beitz, R. Determinants of diet quality. Public Health Nutr. 2004, 7 , 29-37. [CrossRef] [PubMed]

42. Drichoutis, A.C.; Lazaridis, P.; Nayga, R.M., Jr.; Kapsokefalou, M.; Chryssochoidis, G. A theoretical and empirical investigation of nutritional label use. Eur. J. Health Econ. 2008, 9, 293-304. [CrossRef] [PubMed]

43. Roberto, C.A.; Bragg, M.A.; Schwartz, M.B.; Seamans, M.J.; Musicus, A.; Novak, N.; Brownell, K.D. Facts up front versus traffic light food labels: A randomized controlled trial. Am. J. Prev. Med. 2012, 43, 134-141. [CrossRef] [PubMed]

44. Drichoutis, A.C.; Lazaridis, P.; Nayga, R.M., Jr. Nutrition knowledge and consumer use of nutritional food labels. Eur. Rev. Agric. Econ. 2005, 32, 93-118. [CrossRef]

45. Powers, A.R.; Struempler, B.J.; Guarino, A.; Parmer, S.M. Effects of a nutrition education program on the dietary behavior and nutrition knowledge of second-grade and third-grade students. J. Sch. Health 2005, 75, 129-133. [CrossRef] [PubMed]

46. Organization for Economic Co-operation and Development. Obesity and the Economics of Prevention: Fit not Fat; OECD Publishing: Paris, France, 2010. 
47. Gortmaker, S.L.; Swinburn, B.A.; Levy, D.; Carter, R.; Mabry, P.L.; Finegood, D.T.; Huang, T.; Marsh, T.; Moodie, M. Changing the future of obesity: Science, policy, and action. Lancet 2011, 378, 838-847. [CrossRef]

48. Sacks, G.; Veerman, J.L.; Moodie, M.; Swinburn, B. "Traffic-light" nutrition labelling and "junk-food" tax: A modelled comparison of cost-effectiveness for obesity prevention. Int. J. Obes. 2011, 35, 1001-1009. [CrossRef] [PubMed]

49. Hodgkins, C.E.; Raats, M.M.; Fife-Schaw, C.; Peacock, M.; Groppel-Klein, A.; Koenigstorfer, J.; Wasowicz, G.; Stysko-Kunkowska, M.; Gulcan, Y.; Kustepeli, Y.; et al. Guiding healthier food choice: Systematic comparison of four front-of-pack labelling systems and their effect on judgements of product healthiness. Br. J. Nutr. 2015, 113, 1652-1663. [CrossRef] [PubMed]

50. Gorton, D.; Ni, M.C.; Chen, M.H.; Dixon, R. Nutrition labels: A survey of use, understanding and preferences among ethnically diverse shoppers in New Zealand. Public Health Nutr. 2009, 12, 1359-1365. [CrossRef] [PubMed]

51. Helfer, P.; Shultz, T.R. The effects of nutrition labeling on consumer food choice: A psychological experiment and computational model. Ann. N. Y. Acad. Sci. 2014, 1331, 174-185. [CrossRef] [PubMed]

52. Van Herpen, E.; Trijp, H.C. Front-of-pack nutrition labels. Their effect on attention and choices when consumers have varying goals and time constraints. Appetite 2011, 57, 148-160.

53. Kleef, E.V.; Dagevos, H. The growing role of front-of-pack nutrition profile labeling: A consumer perspective on key issues and controversies. Crit. Rev. Food Sci. Nutr. 2015, 55, 291-303. [CrossRef] [PubMed]

54. Lobstein, T.; Davies, S. Defining and labelling "healthy" and "unhealthy" food. Public Health Nutr. 2009, 12, 331-340. [CrossRef] [PubMed]

55. EUFIC. Pan-European Consumer Research on In-Store Observation, Understanding \& Use of Nutrition Information on Food Labels, Combined with Assessing Nutrition Knowledge. European Food Information Council: Brussels, Belgium, 2009.

56. Escalon, H.; Bossard, C.; Beck, F. Baromètre Nutrition-Santé 2008-Perceptions, Connaissances et Attitudes en Matière D'alimentation. Available online: http://www.inpes.fr/Barometres/ barometre-sante-nutrition-2008/pdf/consommations-et-habitudes.pdf (accessed on 20 March 2015).

57. Andreeva, V.A.; Salanave, B.; Castetbon, K.; Deschamps, V.; Vernay, M.; Kesse-Guyot, E.; Hercberg, S. Comparison of the sociodemographic characteristics of the large NutriNet-Sante e-cohort with French Census data: The issue of volunteer bias revisited. J. Epidemiol. Community Health 2015, 69, 893-898. [CrossRef] [PubMed]

58. Rayner, M.; Boaz, A.; Higginson, C. Consumer use of health-related endorsements on food labels in the United Kingdom and Australia. J. Nutr. Educ. 2001, 33, 24-30. [CrossRef]

59. Sacks, G.; Rayner, M.; Swinburn, B. Impact of front-of-pack "traffic-light" nutrition labelling on consumer food purchases in the UK. Health Promot. Int. 2009, 24, 344-352. [CrossRef] [PubMed] 
60. Vyth, E.L.; Steenhuis, I.H.; Vlot, J.A.; Wulp, A.; Hogenes, M.G.; Looije, D.H.; Brug, J.; Seidell, J.C. Actual use of a front-of-pack nutrition logo in the supermarket: Consumers' motives in food choice. Public Health Nutr. 2010, 13, 1882-1889. [CrossRef] [PubMed]

(C) 2015 by the authors; licensee MDPI, Basel, Switzerland. This article is an open access article distributed under the terms and conditions of the Creative Commons Attribution license (http://creativecommons.org/licenses/by/4.0/). 